\title{
Small angle x-ray scattering study on role of trapped entanglements in structure of swollen end-linked poly(dimethylsiloxane) networks
}

$\operatorname{AUTHOR}(S)$ :

Kawamura, T; Urayama, K; Kohjiya, S

\section{CITATION:}

Kawamura, T ... [et al]. Small angle x-ray scattering study on role of trapped entanglements in structure of swollen end-linked poly(dimethylsiloxane) networks. JOURNAL OF CHEMICAL PHYSICS 2000, 112(20): 9105-9111

\section{ISSUE DATE:}

2000-05-22

URL:

http://hdl.handle.net/2433/39725

\section{RIGHT:}

Copyright 2000 American Institute of Physics. This article may be downloaded for personal use only. Any other use requires prior permission of the author and the American Institute of Physics. 


\title{
Small angle $x$-ray scattering study on role of trapped entanglements in structure of swollen end-linked poly(dimethylsiloxane) networks
}

\author{
Takanobu Kawamura, Kenji Urayama, ${ }^{\text {a) }}$ and Shinzo Kohjiya \\ Institute for Chemical Research, Kyoto University, Uji, Kyoto-fu, 611-0011, Japan
}

(Received 5 October 1999; accepted 2 March 2000)

\begin{abstract}
Amorphous structure of swollen end-linked poly(dimethylsiloxane) (PDMS) network has been investigated by means of small angle x-ray scattering (SAXS) technique as a function of molecular mass of precursor PDMS $\left(M_{p}\right)$ and junction (cross-link) functionality. The $M_{p}$ dependence of the SAXS profiles for tetra-functional PDMS networks shows a striking crossover at around $M_{p}$ $\approx M_{c}$ where $M_{c}$ represents the critical molecular mass to form entanglement couplings in the PDMS melt. These results strongly suggest that structure of swollen end-linked networks significantly depends on whether precursor chains are well entangled or not before end-linking, and that when precursor chain is long enough to form entanglement couplings, trapped entanglements formed in cross-linking (instead of cross-links) play a major role in elementary mesh of the resulting networks. For the networks of $M_{p} \gg M_{c}$, the correlation length for the network structure is fairly small and comparable to that of dynamic density fluctuation for the solutions of the precursor polymer, and in addition, no appreciable effect of junction functionality on long-range network structure is observed. These observations imply that the contribution of cross-link to static heterogeneity in network structure is screened by trapped entanglement dominant in number. (c) 2000 American Institute of Physics. [S0021-9606(00)51420-2]
\end{abstract}

\section{INTRODUCTION}

Amorphous structure of polymer gels has attracted much attention of physicists and chemists since some small angle $\mathrm{x}$-ray and neutron scattering (SAXS and SANS, respectively) studies revealed that polymer gel exhibits an excess scattering in small angle region relative to solution with the same polymer content. ${ }^{1}$ The excess scattering implies the existence of a spatial long-range structure specific to polymer gel. The origin of the long-range structure in gel has been attributed to some static frozen heterogeneity formed by cross-linking. At the initial stage of research on gel structure, polymer gels prepared by random cross-linking, having network chains with a broad size distribution, were extensively investigated and the static frozen heterogeneity was considered to originate from spatial heterogeneous distribution of crosslinks. ${ }^{1-4}$ Subsequently, it was reported ${ }^{2,5-10}$ that end-linked networks composed of network chains of fairly uniform size, also showed excess scattering as in the case of randomly cross-linked networks. The end-linked networks are prepared by end-linking precursor polymeric chains with functional groups at both ends by means of a multifunctional reagent (cross-linker), so that size of the resulting network chain becomes equivalent to that of precursor chains under complete progress of end-linking reaction. The origin of the longrange structure for end-linked networks has remained unclear because the concept of spatial heterogeneous distribution of cross-links used for a randomly cross-linked network is not applicable to end-linked networks composed of network chains with fairly uniform size. Recently, Bastide et al. ${ }^{1,9}$

\footnotetext{
a) Author to whom correspondence should be addressed. Electronic mail: urayama@scl.kyoto-u.ac.jp
}

have proposed the shortest closed circuit (SCC) model for the long-range structure in end-linked networks. The SCC model attributes the static frozen heterogeneity to size distribution of the SCCs (like "pores" in network) originating from fluctuation in connectivity of the network chains at cross-link (cf. Fig. 40 in Ref. 1, Fig. 6 in Ref. 9, or Fig. 7 in Ref. 10). The SCC model predicts that even if size of network chains is uniform, the SCCs with different sizes are inevitably formed by end-linking, and that the size distribution of the SCCs becomes more noticeable by swelling. The SCC model qualitatively explains the static frozen heterogeneity formed by end-linking, and the SAXS and SANS data for end-linked polydimethylsiloxane (PDMS) networks with unimodal $^{9}$ and bimodal ${ }^{10}$ size distributions of network chains between adjacent cross-links were interpreted on the basis of the SCC model.

The end-linking method has been widely accepted as an approach to prepare a model polymer network whose topological characteristics such as size, size distribution of network chain, and junction (cross-link) functionality can be controlled during preparation. ${ }^{11,12}$ The end-linking method has contributed significantly to fundamental research of rubber elasticity, especially, on the role of trapped entanglement in rubber elasticity. ${ }^{11-17}$ The trapped entanglement is the entanglement of different network chains fixed by cross-links, and it cannot be disentangled permanently due to topological constraints. In contrast to that, temporary entanglement exhibits a finite disentanglement time. The significant contribution of trapped entanglements to network elasticity has been demonstrated on the basis of experimental results on equilibrium elastic modulus and degree of equilibrium swelling for end-linked rubbery networks, ${ }^{12-17}$ though some researchers 
have argued that trapped entanglements suppress thermal fluctuations of cross-links without additionally contributing to elastic modulus. ${ }^{11}$

The role of trapped entanglements in swollen network structure still remains controversial. The original $c^{*}$ theorem postulates complete disinterpenetration of network strands in the equilibrium swollen state without considering the contribution of trapped entanglements to the elementary mesh of swollen network structure. ${ }^{18}$ For example, in the case of an end-linked network, according to the original $c^{*}$ theorem, the elementary mesh corresponds to the precursor chain itself. On the other hand, some researchers have argued that trapped entanglements play a significant or major role in the elementary mesh of swollen networks on the basis of experimental results on the equilibrium degree of swelling and elastic modulus in the swollen state. ${ }^{12-17,19}$ They consider that trapped entanglements significantly contribute to the elementary mesh in swollen network together with cross-links, i.e., a significant degree of overlapping of network strands exists in the swollen state. The dependence of various physical properties on the degree of equilibrium swelling has been reported to obey the predictions of the original $c^{*}$ theorem, ${ }^{8,13,15-17,20}$ which has often been considered as experimental evidence for the validity of the original $c^{*}$ theorem. It has been shown, however, that the same theoretical predictions are derived from a theory considering contribution of trapped entanglements to elementary mesh. ${ }^{15,16,19}$

In this study, we have investigated SAXS of swollen end-linked poly(dimethylsiloxane) (PDMS) networks as a function of the size of precursor PDMS and junction functionality. The degree of entanglement before end-linking as well as the distance between adjacent cross-links have been extensively altered by using precursor PDMS widely varying in molecular mass $(2850 \sim 184000)$. Since the elementary unit of the network structure is the elementary mesh, the dependence of scattering profiles on the size of the precursor chain should provide significant information about the role of trapped entanglements. The effect of junction functionality on network structure has also been examined to elucidate the origin of static heterogeneity formed by end-linking as well as the determinant of elementary mesh.

\section{EXPERIMENT}

\section{A. Precursor PDMS}

The bifunctional precursor PDMS with relatively narrow molecular size distribution was obtained by fractionation of vinyl- or methacryloyl $\left(\mathrm{CH}_{2}=\mathrm{C}\left(\mathrm{CH}_{3}\right) \mathrm{COO}\right.$ - $)$-terminated PDMS (Gelest Inc. or Shinetsu Co., respectively) with a mixture of 2-butanone and methanol. The number- and weight-average molecular masses $\left(M_{n}\right.$ and $M_{w}$, respectively) of the precursor PDMS were determined by gel permeation chromatography (GPC). The chromatograms were obtained with Shimazu GPC LC-6A equipped with a Shimpack GPC80MC column at $54{ }^{\circ} \mathrm{C}$ using chloroform as solvent. Under this condition, molecular mass of PDMS is acquired from an elution time curve for standard poly(styrene) samples without any further calculations. ${ }^{21}$ The $M_{n}, M_{w}$, and $M_{w} / M_{n}$ data for each precursor chain are listed in Table
TABLE I. Number- and weight-average molecular masses of precursor PDMS ( $M_{n}$ and $M_{w}$, respectively).

\begin{tabular}{crrc}
\hline \hline Precursor PDMS & \multicolumn{1}{c}{$M_{n}$} & \multicolumn{1}{c}{$M_{w}$} & $M_{w} / M_{n}$ \\
\hline V184 & 184000 & 224000 & 1.22 \\
V112 & 112000 & 137000 & 1.22 \\
V42 & 42400 & 54700 & 1.29 \\
V47 & 46600 & 89500 & 1.92 \\
V24 & 23800 & 31400 & 1.32 \\
X13 & 12800 & 15700 & 1.23 \\
X7 & 7290 & 9040 & 1.24 \\
X3 & 2850 & 3680 & 1.29 \\
\hline \hline
\end{tabular}

I. The character " $V$ " or " $X$ " in sample codes of the precursor PDMS represents the functional group at the ends, vinyl and methacryloyl group, respectively.

\section{B. Sample preparation}

Triskis(dimethylsiloxy)silane, tetrakis(dimethylsiloxy)silane, and random copolymer composed of dimethylsiloxane and hydromethylsiloxane units (DMS-HMS) were employed as tri-, tetra-, and 25-functional cross-linker, respectively. $M_{n}$ and the number of $\mathrm{Si}-\mathrm{H}$ sites of DMS-HMS were estimated as 6000 and 25 by GPC and ${ }^{1} \mathrm{H}-\mathrm{NMR}$ (JEOL JNM-AL 400), respectively. A platina chloric acid dissolved in isopropanol (Spier's catalyst) was used as catalyst for hydrosilylation between the silane hydrogen and vinyl or methacryloyl group.

The mixtures containing precursor PDMS, cross-linker, and catalyst were poured into a Teflon mold. The molar ratios of cross-linker to precursor PDMS and to catalyst for each sample were adjusted in such a way that the amount of unreacted material in the resulting gel was minimum (the method proposed by Patel et al. .). ${ }^{14-16}$ The ratios of silane hydrogens to vinyl groups $(r)$ for each sample are shown in Table II. The numerals before and after "F" in the gel

TABLE II. The ratios of silane hydrogen to vinyl group $(r)$, volume fractions of networks $\left(\phi_{e}\right)$, and correlation lengths $(\Xi)$ for equilibrium swollen PDMS networks.

\begin{tabular}{rclc}
\hline \hline Sample & $r$ & \multicolumn{1}{c}{$\phi_{e}$} & $\Xi / \AA$ \\
\hline 184F04 & 1.5 & 0.0874 & 22.0 \\
112F04 & 1.4 & 0.108 & 20.2 \\
42F04 & 1.5 & 0.140 & 27.8 \\
24F04 & 1.8 & 0.230 & 23.7 \\
13F04 & 1.7 & 0.146 & 48.5 \\
7F04 & 1.6 & 0.139 & 49.0 \\
3F04 & 1.8 & 0.234 & 35.1 \\
184F25 & 1.5 & 0.132 & 20.5 \\
184F03 & 1.5 & 0.0443 & 28.0 \\
42F25 & 1.5 & 0.216 & 26.0 \\
42F03 & 1.5 & 0.132 & 45.0 \\
24F25 & 1.5 & 0.306 & 22.0 \\
24F03 & 1.8 & 0.128 & $301^{\mathrm{a}}$ \\
47F04 & 1.7 & 0.111 & 27.5 \\
\hline \hline
\end{tabular}

${ }^{a}$ The value includes large error due to the absence of quasisaturation of scattering intensity essential for curve fitting of Eq. (1). 
sample codes represent, respectively, $M_{n}$ of precursor PDMS and functionality of the cross-linker. The cross-linking reaction was carried out at $80^{\circ} \mathrm{C}$ for three days.

The resulting network samples were swollen in octane until equilibrium swelling was achieved. The octane was changed every day to remove soluble components. The weight fractions of the soluble components $\left(w_{\text {sol }}\right)$ for all the gel samples, except ones using V184, were less than $8 \%$. The values of $w_{\text {sol }}$ for the gels using the longest precursor chain V184 were ca. $10 \%$, and $w_{\text {sol }}$ for the samples using V24 were fairly small (ca. 1\%). The volume fraction of PDMS network in the equilibrium swelling state $\left(\phi_{e}\right)$ was calculated from the weight in the fully swollen and in the dry state (after extraction of the soluble components). $\phi_{e}$ of each sample is tabulated in Table II.

\section{SAXS measurement}

The SAXS measurements were performed at the beam station BL-10C in the Photon Factory in Institute of Materials Structure Science (Tsukuba, Japan). An incident x-ray from synchrotron radiation was monochromatized to $\lambda=1.488 \AA$ with a double-crystal monochromator. The monochromatized $\mathrm{x}$-ray was focused to sample cell position with a bent focusing mirror. The fully swollen gel samples were placed in a cell with mica windows on the both sides. The thickness of the cell was $2 \mathrm{~mm}$. The intensity of scattered $\mathrm{x}$-ray was measured by a one-dimensional positionsensitive proportional counter. The data calibration was conducted by subtracting the scattering of empty cell and octane from that of the gel samples.

\section{RESULTS}

\section{A. Effect of size of precursor chain}

Figure 1 shows scattering profiles of the fully swollen tetra-functional PDMS networks as a function of size of the precursor PDMS. In the figure, $I$ and $q$ is the scattering intensity and magnitude of scattering vector, respectively. For comparison of the gels with different degrees of swelling, $I$ of each sample was normalized by the conventional method, i.e., divided by $\phi_{e} .{ }^{1}$ The scattering profile of the solution of uncrosslinked V184, having the same polymer content as for the gel 184F04, is also shown in the same figure. It is found that the gel 184F04 exhibits excess scattering in small $q$ region relative to the solution of the uncross-linked precursor polymer, which implies the existence of a spatial long-range structure specific to the gel, as in the case of various gels reported earlier. ${ }^{1-10}$ In comparison of the scattering profiles of each gel, a striking difference is observed between the two groups, the gels of $M_{p} \geqslant 23800$ and $M_{p} \leqslant 12800$, where $M_{p}$ is the number-average molecular mass of precursor PDMS. The $M_{p}$ dependence of the scattering curves among each group is much weaker relative to the large difference between the gels of $M_{p}=12800$ and 23800 . The scattering curves of the networks of $M_{p} \geqslant 23800$ show quasiplateau region at small $q$, while $I$ of $M_{p} \leqslant 12800$ does not fully saturate and the curves show a shoulder. A definite level off of $I$ for the networks of $M_{p} \leqslant 12800$ presumably occurs at smaller $q$. In large $q$ region, the slopes of $\log I$ vs $\log q$ plots

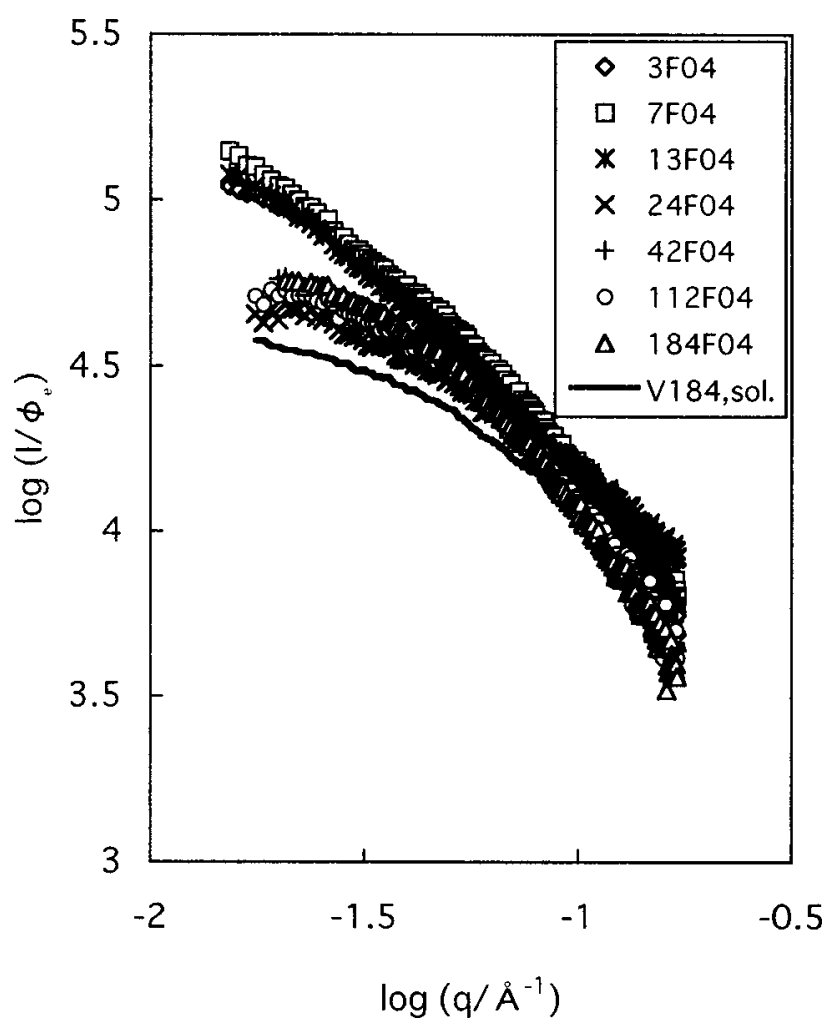

FIG. 1. Normalized scattering profiles for swollen tetra-functional PDMS networks as a function of size of precursor PDMS. Scattering curve of uncrosslinked V184 solution, having same polymer concentration as for $184 \mathrm{~F} 04$, is also shown. The scattering intensities are normalized by $\phi_{e}$.

are $-1.5 \sim-2.0$, which might be related to spatial correlation of an isolated polymer chain under excluded volume effect $\left(I \sim q^{-5 / 3}\right)$ and in the unperturbed state $\left(I \sim q^{-2}\right)$.

Figure 2 shows the plots of $I^{-1}$ vs $q^{2}$ in the $q$ region of

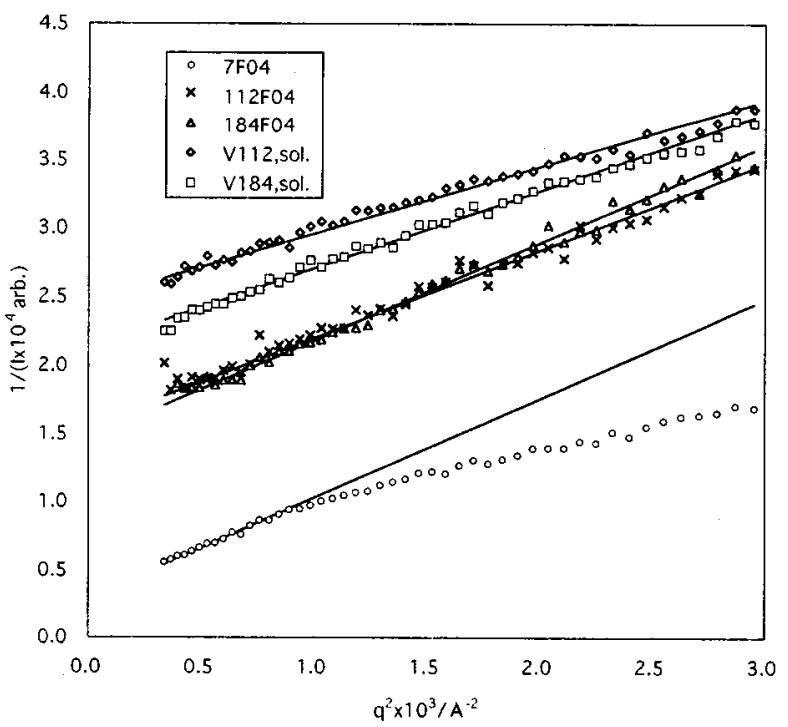

FIG. 2. The plots of $I^{-1}$ vs $q^{2}$ in small $q$ region for the gels 184F04, 112F04, 7F04, and the solutions of uncross-linked PDMS V184 and V112. The polymer volume fractions in the solutions are the same as those in the correponding gels. The solid lines represent linear extrapolation toward $q$ $=0$. 
TABLE III. Volume fractions of polymer $(\phi)$ and correlation lengths $(\xi)$ for solutions of uncross-linked precursor PDMS.

\begin{tabular}{ccc}
\hline \hline Sample & $\phi$ & $\xi / \AA$ \\
\hline V184 & 0.0874 & 16.3 \\
V112 & 0.108 & 14.1 \\
V42 & 0.140 & 11.2 \\
V24 & 0.230 & 7.30 \\
X13 & 0.146 & 9.60 \\
\hline
\end{tabular}

$q<5.5 \times 10^{-2} \AA^{-1}$ for tetrafunctional gels $184 \mathrm{~F} 04,112 \mathrm{~F} 04$, and 7F04. The data of the solutions of uncross-linked V112 and V184, having the same polymer content as for the corresponding gel, are also shown in the figure. Linear relations between $I^{-1}$ vs $q^{2}$ are observed in the small $q$ region for each sample; in the region $q>3.2 \times 10^{-2} \AA^{-1}$ the data for 7F04 deviate from the linear relationship. Such linear relations suggest that the scattering data in the small $q$ region obey the following Ornstein-Zernicke scattering function:

$$
I=\frac{I_{0}}{1+\Xi^{2} q^{2}}
$$

where $\Xi$ and $I_{0}$ is correlation length and scattering intensity at $q=0$, respectively. The scattering data of all other networks exhibit linear relation in the small $q$ region, and $\Xi$ for each sample was evaluated by linear regression toward $q$ $=0$. The values of $\Xi$ for each gel sample are listed in Table II. It is found that $\Xi$ for the gels of $M_{p} \geqslant 23800$ are comparable, and they are smaller than $\Xi$ of $M_{p} \leqslant 12800$. The correlation lengths for the solutions $(\xi)$ were obtained by the same procedure, and the values of $\xi$ for samples V184, V112, V42, V24, and V13 are shown in Table III. We distinguish correlation lengths in the small $q$ region between polymer solution $(\xi)$ and gel $(\Xi)$ because of their different physical origin: $\xi$ is related to dynamic fluctuation of polymer concentration, ${ }^{1}$ while $\Xi$ is generally dominated by static nonuniformity.

Several researchers have proposed different types of scattering functions to describe the whole scattering spectra of polymer gels. Most of them consist of the sum of two different functions: a Lorentzian and squared Lorentzian; ${ }^{22}$ a Lorentzian and stretched exponential, ${ }^{23}$ a Lorenzian and Gaussian $;{ }^{24}$ another two complex functions. ${ }^{4}$ It is to be noted, however, that none of them can reproduce satisfactorily and consistently the experimental scattering patterns over the entire range of $q$ for all samples in the present study.

Figure 3 shows the comparison of scattering profiles for the two gels (42F04 and 47F04) whose precursor chains have comparable $M_{n}$ (ca. 45000) but different degrees of size distribution $\left(M_{w} / M_{n}=1.29\right.$ and 1.92 , respectively). The scattering profiles for 47F04 and 42F04 agree well all over the $q$ region examined here.

\section{B. Effect of junction functionality}

Figure 4 shows the scattering profiles of the gels whose precursor chains are V24, V42, and V184 as a function of junction functionality. In this figure, the scattering profiles for the gels of V42 and V184 are shifted vertically in order to

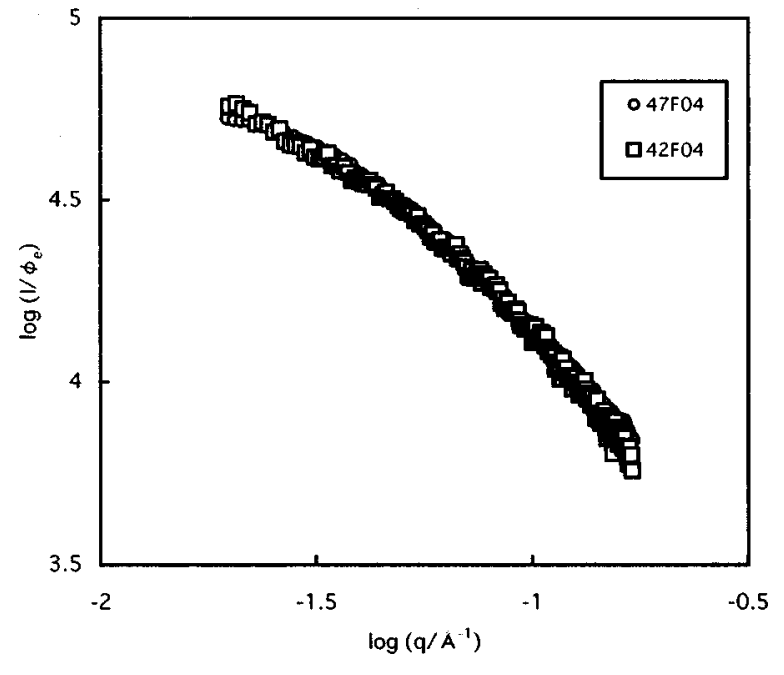

FIG. 3. Comparison of normalized scattering profiles for two swollen tetrafunctional PDMS networks whose precursor PDMS have comparable number-average molecular mass (ca. 45 000) but different size distributions $\left(M_{w} / M_{n}=1.29\right.$ and 1.92). The scattering intensities are normalized by $\phi_{e}$.

avoid overlapping. It can be noticed that the functionality effect on long-range structure (in small $q$ region) is recognizable for the gels of V24 and V42, while it is not appreciable for the gels of V184. For the gels of V24 and V42, the scattering curves of tetra- and 25 -functional networks show quasiplateau region at small $q$, while $I$ of the trifunctional network was not fully saturated in the small $q$ region exam-

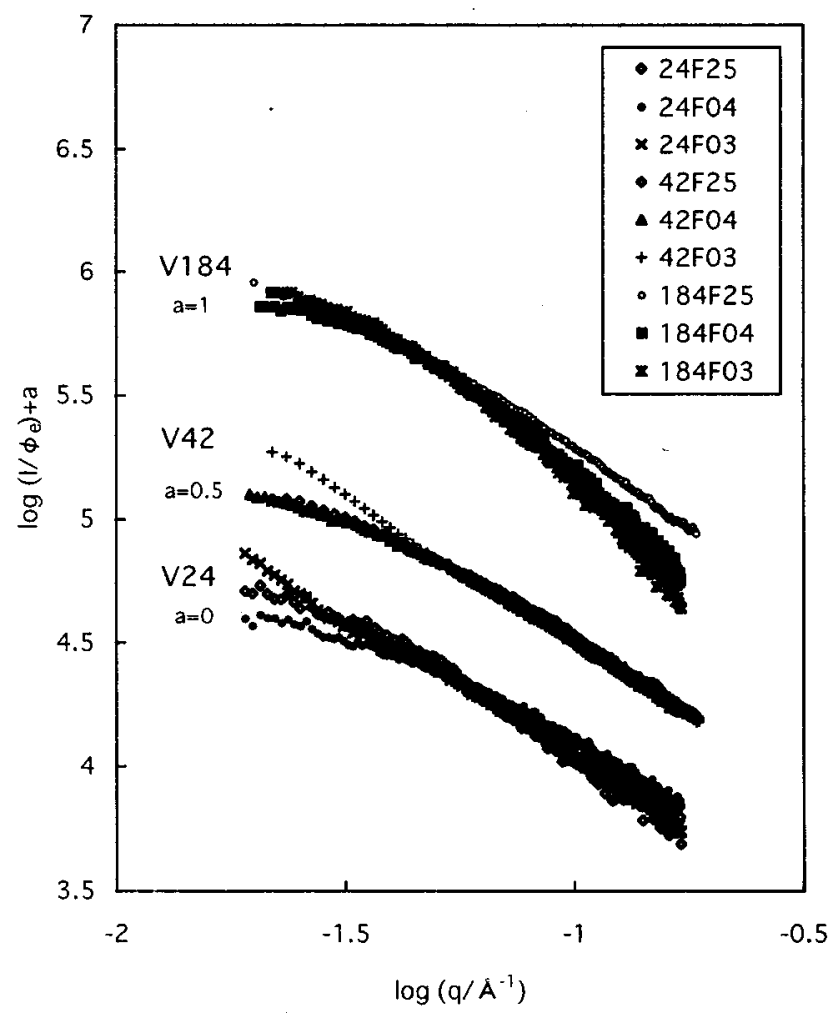

FIG. 4. Normalized scattering profiles of swollen PDMS networks whose precursor chain is V24, V42, V184 as a function of junction functionality. The scattering intensities are normalized by $\phi_{e}$. The curves of the gels of V42 and V184 are vertically shifted to avoid overlapping. 
ined here. On the other hand, $I$ for the gels of V184 level off at small $q$ regardless of the functionality. The values of $\Xi$ for these samples were evaluated by fitting Eq. (1) to the data at small $q$, and listed in Table II. The value of $\Xi$ of $24 \mathrm{~F} 03$ inevitably includes large error due to the absence of quasisaturation of $I$ in the $q$ range examined here. However, it is evident that 24F03 has larger $\Xi$ than $24 \mathrm{~F} 04$ and 24F25. It is found that $\Xi$ for the trifunctional networks increases with decreasing $M_{p}$, while $\Xi$ for the tetra- and 25-functional networks are not sensitive to $M_{p}$.

It can be observed in high $q$ region that the $q$ dependence of $I$ for $184 \mathrm{~F} 25$ is weaker than those for 184F04 and 184F03, but this difference in short-range structure should be owing to the high polymer concentration of $184 \mathrm{~F} 25$ which is rather higher than those of 184F04 and 184F03, and close to that of the gels of V42. Actually, the $q$ dependence of $I$ of $184 \mathrm{~F} 25$ in the high $q$ region is similar to that of the gels of V42 with comparable $\phi_{e}$.

\section{DISCUSSION}

As can be seen in Fig. 1, the scattering profiles of the end-linked tetra-functional PDMS gels can be roughly categorized into the two groups, $M_{p} \geqslant 23800$ and with $M_{p}$ $\leqslant 12800$. It can be noticed that the reported value ${ }^{25}$ of $M_{c}$ $(=16600)$ for PDMS is located in the region $23800>M$ $>12800$ where the striking crossover is observed. Here, $M_{c}$ is the critical molecular mass of polymer chain to form entanglement couplings in an uncross-linked polymer melt. This result strongly suggests that whether precursor chain is well entangled or not before end-linking is a dominant factor for structure of the resulting polymer networks in the swollen state. Since the precursor chains of $M_{p} \geqslant 23800\left(>M_{c}\right)$ are well entangled before end-linking, the number of trapped entanglements formed in cross-linking should greatly exceed the number of cross-links in the resulting networks. The SAXS profiles not sensitive to $M_{p}$ in the region $M_{p}$ $\geqslant 23800$ imply that when the precursor chain is long enough to form entanglement couplings, the elementary mesh of the resulting networks is mainly governed by neighboring trapped entanglements, instead of neighboring cross-links. It is to be noted that spatial distance between neighboring entanglements is independent of molecular mass of polymer chains, as clearly revealed by a specific plateau elastic modulus of uncross-linked polymer melt irrespective of molecular mass. ${ }^{26}$ The molecular mass between neighboring entanglements $\left(M_{e}\right)$ for PDMS was estimated as 8100 from plateau modulus of uncross-linked PDMS melt. ${ }^{26}$ The character of trapped entanglements as scattering center may not be same as that of chemical cross-links due to difference in molecular mobility. However, the striking crossover around $M_{p} \approx M_{c}$, as well as scattering profiles independent of $M_{p}$ in the region of $M_{p}>M_{c}$ suggest that trapped entanglements act as effective scattering centers. The character of trapped entanglements as scattering centers may be qualitatively similar to that of overlapping points of entangled polymer chains constituting hypothetical transient network in semidilute polymer solution.

It is to be emphasized that if the elementary mesh is entirely controlled by cross-links, the scattering profiles must depend primarily on $M_{p}$ according to change in elementary mesh size against $M_{p}$.

Patel et al. ${ }^{14}$ observed pronounced effect of trapped entanglements on elastic modulus in end-linked PDMS networks of $M_{p}>M_{c}$. They investigated the $M_{p}$ dependence of equilibrium elastic modulus of dry PDMS networks endlinked in the bulk state. The elastic moduli of the networks in the region of $M_{p}>M_{c}$ were almost constant and close to plateau modulus of the uncross-linked PDMS melt. Their results suggests that the length of elastically effective strands is governed by the distance between neighboring trapped entanglements for the networks whose precursor chains are well-entangled before cross-linking, and that molecular mass between neighboring trapped entanglements is equivalent to that between neighboring temporary entanglements in the uncross-linked polymer melt. They also showed ${ }^{14}$ that trapped entanglements significantly contribute to network elasticity even in the swollen state. The observation in their study is an independent experimental evidence to support the idea that trapped entanglements play a dominant role in elementary network mesh for the swollen networks of $M_{p}$ $>M_{c}$.

As shown in our previous article, ${ }^{16}$ even the networks prepared from an oligomeric precursor chain of $M_{p}<M_{c}$ have a nonnegligible amount of trapped entanglements due to a finite overlapping of the precursor chain before endlinking. The number of trapped entanglements in such networks, however, is comparable or smaller than the number of cross-links, in contrast to networks whose precursor chains are well entangled before cross-linking. Accordingly, for networks prepared from oligomeric precursor chains, effect of the number of cross-links, i.e., the size of the precursor chain can influence elementary mesh. The fraction of cross-links in the total number of "effective" junctions (cross-links plus trapped entanglements) decreases with increasing $M_{p}$, and is critically reduced around $M_{p} \approx M_{c}$ because of formation of temporary entanglement couplings before cross-linking. The major origin of the remarkable crossover in the scattering profiles in $23800>M_{p}>12800$ is attributable to the change in determinant of elementary mesh.

In Fig. 3 no appreciable effect of size distribution of precursor chain on scattering profiles is observed. It is to be noticed that the precursor chains used here have the average molecular mass exceeding $M_{c}$. This result shows that the effect of size distribution of precursor chain on swollen network structure is negligibly small, when precursor chains are long enough to form entanglement couplings before endlinking. This result is another evidence showing that elementary mesh is mainly controlled by trapped entanglements, instead of cross-links, for such the networks. As shown in our previous study, ${ }^{10}$ however, a bimodal size distribution of precursor chains with a fraction of very short chain $\left(M_{p}\right.$ $\approx 4000$ ) significantly influences the structure of the resulting network, even if the average molecular size is larger than $M_{c}$. In the case of a mixture of precursor chains containing very short chain, some specific effects of very short precursor chain on network structure can appear.

Figure 5 shows the double logarithmic plots of correlation length ( $\Xi)$ against $M_{p}$ for the tetra-functional networks. 


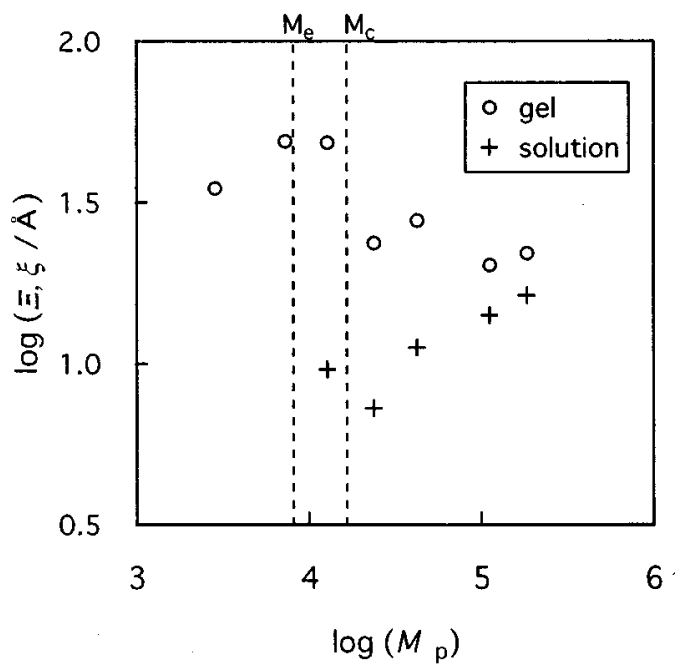

FIG. 5. The $M_{p}$ dependence of $\Xi$ and $\xi$ for swollen tetra-functional PDMS networks and solutions of precursor PDMS, respectively. The polymer volume fractions in gel and solution of each precursor PDMS are identical. $M_{p}, M_{e}, M_{c}$ represents the number-average molecular mass of precursor PDMS, the molecular mass between neighboring entanglements for PDMS $(8,100)$, and the critical molecular mass to form entanglement couplings in uncrosslinked PDMS melt (16600), respectively. $\Xi$ and $\xi$ are correlation lengths for gel and solution, respectively.

It can be seen that $\Xi$ remarkably changes around $M_{c}$, while $\Xi$ is almost constant in the region $M_{p}>M_{c}$ regardless of $M_{p}$. The constancy of $\Xi$ in the region $M_{p}>M_{c}$ is expected from the independence of the scattering profiles on $M_{p}$. Since elementary mesh size is governed by the distance between neighboring trapped entanglements (independent of $M_{p}$ ), the networks of $M_{p}>M_{c}$ have almost the same structure, namely, a structure of "entanglement-dominant network." It is to be emphasized again that if elementary mesh size is the distance between neighboring cross-links, $\Xi$ should depend on $M_{p}$ as a result of change in elementary mesh size against $M_{p}$.

Figure 5 also includes the data of correlation length for dynamic density fluctuation $(\xi)$ for solutions of the uncrosslinked precursor chains having the same polymer volume fraction as for the corresponding gel. It can be noticed that the difference between $\xi$ and $\Xi$ decreases with increasing $M_{p}$. In addition, $\Xi$ of the entanglement-dominant networks (of $M_{p} \gg M_{c}$ ) are found to be fairly small and comparable to $\xi$ of the corresponding solutions in spite of the high degree of swelling near or over 10. It has been observed in various gels that the static heterogeneity is more pronounced as the degree of swelling increases. ${ }^{1,26,27}$ It is to be also noticed that 7F04 and 3F04 show larger $\Xi$ than the entanglementdominant networks, though the elementary mesh sizes of 7F04 and 3F04 (approximated by $M_{p}$ ) are smaller than those of the entanglement-dominant networks $\left(M_{e} \approx 8100\right)$. This is contrary to the simple similarity in network structure postulating that $\Xi$ (a spatial extent characterizing spatial higherorder structure) may increase with the increase in elementary mesh size. Such unexpectedly small degree of spatial heterogeneity in the entanglement-dominant networks appears to suggest that trapped entanglements have a screening effect on the contribution of cross-links to heterogeneous network structure. It is widely accepted that cross-links play an essential role in static frozen heterogeneity of network structure regardless of the type of cross-linking. ${ }^{1}$ The contribution of cross-link on network structure is expected to be more highly screened due to reduction in concentration of cross-link, as the fraction of trapped entanglements in total effective junctions increases (i.e., size of precursor chain is larger). The reduction of the difference $(\Xi-\xi)$ against $M_{p}$ is likely to be interpreted as a result of the increase of screening due to the increasing fraction of trapped entanglements. The magnitude of $\Xi$ of the entanglement-dominant networks is ca. $25 \AA$ which is much smaller than the typical reported values $(>50$ $\AA$ ) of correlation lengths for static frozen heterogeneity in various polymer gels. ${ }^{1}$ The earlier studies, ${ }^{28}$ reporting larger values of $\Xi$, concerned the gels with relatively high fraction of cross-links: The gels were prepared by monomer polymerization, or end-linking precursor chains which are not well entangled, or by introducing some cross-links into long precursor chains by random cross-linking. In such gels, the screening effect by trapped entanglement may be so small that the contribution of cross-links to static heterogeneity can be recognizable.

It can be seen in Fig. 4 that the effects of junction functionality on long-range network structure are more distinct as the size of precursor chain decreases. The gels of V184 which is highly entangled before cross-linking show no discernible differences in the scattering profiles in small $q$ region between different functionalities. As in the case of the $M_{p}$ dependence of $(\Xi-\xi)$, no appreciable functionality effect for the gels of V184 also implies screening of contribution of cross-link to network structure by trapped entanglements dominant in number.

It is found in Table II that for the gels of V24 and V42, $\Xi$ of the trifunctional networks are definitely larger than $\Xi$ of the corresponding tetra- and 25-functional networks, though $\Xi$ of the tetra- and 25-functional networks are comparable. According to the concept of the SCC model, ${ }^{1,9}$ the average size of the shortest closed circuits (SCC), i.e., $\Xi$, increases with increase in fluctuation of connectivity at network chains at cross-link. The suppression of fluctuation in connectivity is expected to increase with increase in junction functionality: The probability to form large SCC (like pore) by end-linking is reduced by increasing the degree of branching at cross-link. This expectation agrees with the result that $\Xi$ of the trifunctional (the lowest functional) networks 24F03 and 42F03 are larger than those of the corresponding higher functional networks.

\section{CONCLUSIONS}

The SAXS of end-linked PDMS networks in the swollen state has been investigated as a function of molecular mass of precursor PDMS $\left(M_{p}\right)$ and junction functionality. The $M_{p}$ dependence of the SAXS profiles of swollen tetra-functional PDMS networks shows a striking crossover at $M_{p} \approx M_{c}$, and the scattering profiles are almost independent of $M_{p}$ in the region $M_{p}>M_{c}$. No appreciable effect of size distribution of precursor chain on scattering curves is observed for the networks of $M_{p}>M_{c}$. These results strongly suggest that the 
structure of the end-linked network is significantly influenced by whether precursor chain is well-entangled or not before end-linking, and that trapped entanglements play a major role in the elementary mesh of swollen network structure when the precursor chain is long enough to form entanglements.

For the networks of $M_{p} \gg M_{c}$, the correlation lengths are fairly small and comparable to correlation lengths of dynamic density fluctuation for solutions of the precursor polymers, and in addition, no significant dependence of longrange structure on junction functionality is observed. These results are likely to be interpreted as a result of screening of the contribution of cross-link to network structure by trapped entanglements dominant in number.

For the networks prepared from modestly long precursor chains, the tetra- and 25-functional networks show smaller correlation lengths for static heterogeneity relative to the trifunctional networks. This result is explained by considering the suppression effects of high-functional junction on fluctuation in connectivity of network chains which is a major origin of static nonuniformity in a swollen end-linked network.

\section{ACKNOWLEDGMENTS}

The authors are greatly indebted to Dr. H. Urakawa, Kyoto Institute of Technology, for the SAXS measurements. This work was performed with the approval of the Photon Factory in Institute of Materials Structure Science, Tsukuba, Japan (Proposal No. 98G088). This work is partly supported by a Grant-in-Aid from the Ministry of Education, Science, and Culture of Japan (No. 09750990), and the grants from the Asahi Glass Foundation and the Eno Science Foundation, to all of which K.U. wishes to express his gratitude.

${ }^{1}$ J. Bastide and S. J. Candau, in Physical Properties of Polymer Gels, edited by A. Cohen (Wiley, New York, 1996), and references cited therein.

${ }^{2}$ J. Bastide, F. Boué, and M. Buzier, in Molecular Basis of Polymer Net- works, edited by A. Baumgärtner and C. E. Picot (Springer-Verlag, Berlin, 1989).

${ }^{3}$ E. Geissler, A.-M. Hecht, S. Mallam, F. Horkay, and M. Zrínyi, Makromol. Chem., Macromol. Symp. 40, 101 (1990).

${ }^{4}$ A. N. Falcão, J. S. Pedersen, and K. Mortensen, Macromolecules 26, 5350 (1993).

${ }^{5}$ V. K. Soni and R. S. Stein, Macromolecules 23, 5257 (1990).

${ }^{6}$ E. Mendes, B. Girard, C. Picot, M. Buzier, F. Boué, and J. Bastide, Macromolecules 26, 6873 (1993).

${ }^{7}$ E. Geissler, F. Horkay, and A.-M. Hecht, J. Chem. Phys. 100, 8418 (1994).

${ }^{8}$ M. Shibayama, H. Takahashi, and S. Nomura, Macromolecules 28, 6860 (1995).

${ }^{9}$ C. Rouf-George, J. P. Munch, G. Beinert, F. Iset, A. Pouchelon, J. F. Palierne, F. Boué, and J. Bastide, Polym. Gels and Networks 4, 435 (1996).

${ }^{10}$ K. Urayama, T. Kawamura, Y. Hirata, and S. Kohjiya, Polymer 39, 3827 (1998).

${ }^{11}$ J. E. Mark, Adv. Polym. Sci. 44, 1 (1982).

${ }^{12}$ M. Gottlieb, C. W. Macosko, G. S. Benjamin, K. O. Meyers, and E. W. Merril, Macromolecules 14, 1039 (1981).

${ }^{13}$ S. Candau, A. Peters, and J. Herz, Polymer 22, 1504 (1981).

${ }^{14}$ S. K. Patel, S. Malone, C. Cohen, J. R. Gillmore, and R. H. Colby, Macromolecules 25, 5241 (1994).

${ }^{15}$ K. Urayama and S. Kohjiya, J. Chem. Phys. 104, 3352 (1996).

${ }^{16}$ K. Urayama, T. Kawamura, and S. Kohjiya, J. Chem. Phys. 105, 4833 (1996).

${ }^{17}$ T. Kawamura, K. Urayama, and S. Kohjiya, Nihon Reoroji Gakkaishi 25, 195 (1997).

${ }^{18}$ P.-G. de Gennes, Scaling Concepts in Polymer Physics (Cornell University Press, Ithaca, New York, 1979).

${ }^{19}$ S. P. Obukhov, M. Rubinstein, and R. H. Colby, Macromolecules 27, 3191 (1994).

${ }^{20}$ H. Oikawa and K. Murakami, Macromolecules 24, 1117 (1991).

${ }^{21}$ B. Wang and S. Krause, Macromolecules 20, 2201 (1987).

${ }^{22}$ A. Onuki, J. Phys. II 2, 45 (1992).

${ }^{23}$ F. Horkay, A.-M. Hecht, S. Mallam, E. Geissler, and A.-R. Rennie, Macromolecules 24, 2896 (1991).

${ }^{24}$ A.-M. Hecht, R. Duplessix, and E. Geissler, Macromolecules 18, 2167 (1985)

${ }^{25}$ D. J. Orrah, J. A. Semlyen, and S. B. Ross-Murphy, Polymer 29, 1452 (1988).

${ }^{26}$ J. D. Ferry, Viscoelastic Properties of Polymers, 3rd ed. (Wiley, New York, 1980).

${ }^{27}$ E. Mendes, P. Lindner, M. Buzier, F. Boué, and J. Bastide, Phys. Rev. Lett. 66, 1595 (1991).

${ }^{28}$ E. Geissler, F. Horkay, and A.-M. Hecht, Phys. Rev. Lett. 71, 645 (1993). 